\title{
Lifting effect of sequential autologous fat injection (SAFI) in three patients with small and flat malar bones
}

\author{
Kyoungjin Kang, MD, PhD iD \\ Seoul Cosmetic Surgery Clinic, Busan, Rep. of Korea
}

\begin{abstract}
Various fat grafting techniques are commonly performed to restore the facial volume deficiency in the soft tissue. Generally, the purified fat is evenly injected into the whole layer in the volume deficit area. The sequential autologous fat injection (SAFI) technique is known to have a volumetric lifting effect. In patients with small and flat malar bones, the soft tissue foundation becomes weak; hence, the soft tissue overlying the bone easily sags even in young patients. Therefore, the author tried to apply the SAFI technique to correct the sagging face. The fat was harvested and purified by the Coleman's method, and sequentially injected into each defective region in the superolateral to anteroinferior direction. Furthermore, the fat was injected consequently in the deep, middle, and superficial layers to increase the skeletal support for the soft tissue using the SAFI technique. An increase in the volume in the malar and sub-malar areas and decrease in the volume in the deep nasolabial folds and jowls were simultaneously observed. Moreover, the facial contour changed from square to inverted triangle type with shallow nasolabial folds and lifted appearance of the jowls in these 3 cases. The results indicated that the SAFI technique could be used for volumetric face lifting, because it focuses on the correction of the skeletal deformity in order to improve the soft tissue foundation.
\end{abstract}

Keywords: deep nasolabial fold; facial contour; fat grafting; sagging jowl; volumetric lifting

\section{Introduction}

Fat grafting has been widely performed as a conventional method for the correction of wrinkles, folds, and depressions in the face and body. Several techniques including structural fat grafting [1], fat rebalancing [2], fat autologous muscular injection [3], complement fat grafting [4], and sequential autologous fat injection (SAFI) [5] have been developed. However, no one has tried to improve the skeletal deformity using the fat grafting procedure.

Generally, in the patients with small zygomatic bones, aging manifestations such as deep nasolabial folds and sagging jowls are commonly seen in both young and old patients. This is mainly due to the decreased skeletal support of the soft tissue. An allograft implant has been widely used as a hard material to correct the bone deformity.

Herein, the author first injected the fat supraperiosteally and beneath the ligaments, and then sequentially injected it into the deep and superficial fat layers using the SAFI technique in 3 patients with small symmetric or asymmetric zygomatic bones.

\section{Case report}

The fat harvesting and purification procedures were performed as a modification of the Coleman's method [1]. The purified fat was unevenly and sequentially injected into each

Received November 28, 2019; Revised December 26, 2019; Accepted December 30, 2019

Corresponding author: Kyoungjin Kang

E-mail: mdkjkang@hanmail.net

This is an Open Access article distributed under the terms of the Creative Commons Attribution Non-Commercial License (http://creativecommons.org/licenses/by-nc/4.0), which permits unrestricted non-commercial use, distribution, and reproduction in any medium, provided the original work is properly cited.

Copyright ( 2019 Korean Society of Korean Cosmetic Surgery and Medicine (KSKCS \& KCCS). 
layer, wherein about $60 \%$ volume of the grafted fat was first attached to the periosteal layer for the correction of the skeletal deformity in the zygomatico-maxillary bone, followed by about $30 \%$ and $10 \%$ separately injected into the deep and superficial fat layers for soft tissue volume restoration, respectively. In the area without the bone, the fat was attached to the muscle fascia or injected into the deep fat pad. The fat was also injected in a regular order as shown in Fig. 1 [6]. The grafting was performed under local anesthesia, assisted by intravenous sedation using propofol.

\section{Case 1}

Preoperatively, the malar area appeared smaller and flatter and the nasolabial and labiomandibular folds were longer and deeper on the right side of the face as compared to the left (Fig. $2 \mathrm{~A}, \mathrm{D}, \mathrm{G})$. Compared to the left lower face, the right lower face showed severe sagging appearance with a rounded contour.

Postoperatively, the volumes of the malar and submalar regions were perfectly restored and the nasolabial and labiomandibular folds were shortened and became shallow. Furthermore, the volume of the lateral nasolabial folds and jowls had remarkably reduced. A volume increase in the malar, midcheek furrow, submalar, and lateral cheek areas (Fig. 2D, G) and contour change (concaved) in the mid and lower face (Fig. 2F,

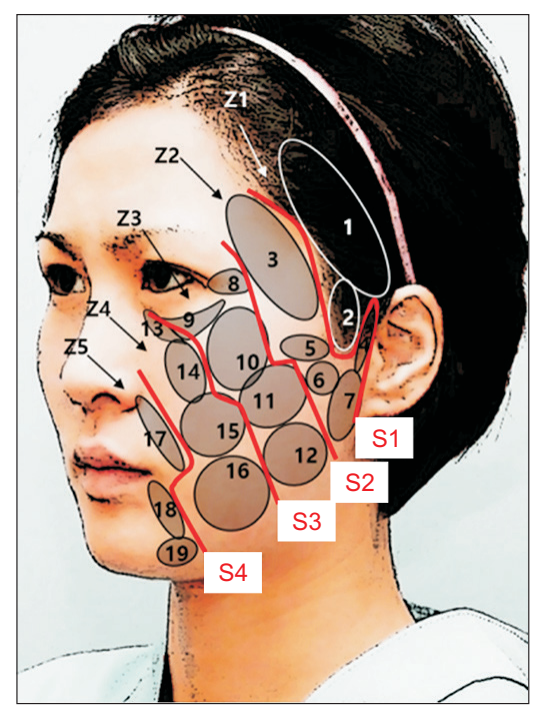

Fig. 1. Sequential autologous fat injection (SAFI) lines, zones, and possible injection areas of SAFI technique. The face is divided into 5 zones by 4 SAFI lines. The purified fat is sequentially injected from Zone 1 to Zone 5, from the upper area to lower area in each zone, and then injected into the deep, middle, and superficial layers in order. The numbers from 1 to 19 refer to the order of the injections. Z1-5, injected zone; S1-4, SAFI line.
I) were observed. The jowl lines became tighter and changed from round to $\mathrm{V}$-shape.

\section{Case 2}

Preoperatively, the patient had tear trough deformities, deep nasolabial folds, and sagging jowls even at a young age (Fig. 3A).

Postoperatively, dramatic volume rebalancing caused by the combination of volume reduction of the jowls and nasolabial folds and volume restorations of the temporal hallow and malar eminence was observed. This contributed to the appearance of the lifted face in the posterosuperior direction (Fig. 3B).

However, the facial contour after settlement of the grafted fat looked very natural with a lifted appearance (Fig. 3C). Despite the $5 \%$ increase in her body weight, the contour was still maintained for up to 1 year (Fig. 3D).

\section{Case 3}

Preoperatively, a bulky pentagonal facial contour with small and flat malar eminences, submalar depressions, and lateral cheeks were observed superolaterally, with deep nasolabial folds and sagging jowls in the anteroinferior direction (Fig. 4A, E).

Postoperatively, the facial contour changed from pentagonal to oval shape, and the face looked smaller and lifted due to the volume increase in the malar and submalar areas and the volume decrease in the jowls and nasolabial folds (Fig. 4B, F).

The nasolabial folds were significantly softened and became shallow, and the size of the jowls was significantly reduced as if the liposuction procedure was performed (Fig. 4B, F). However, the postoperative dynamic changes observed in Fig. 4C and 3D show that the nasolabial folds and jowls almost returned to the same state as observed before (Fig. 4A, E). It occurred simultaneously with noticeable decrease in the volume of the malar, submalar, and lateral cheek regions (Fig. 4C, D, G, H).

\section{Discussion}

The dictionary definition of 'lift' is 'to rise or cause to rise from the ground or another support to a higher place.' The face-lift is defined as a cosmetic surgical operation for tightening sagging skin and smoothing unwanted wrinkles on the face.

Recently, attempts have been made to induce the face-lift effect using fat grafting or man-made filler injections, such as fat rebalancing [2], tower technique, vertical supraperiosteal depot technique [7], and lift-and-fill face-lift technique [8]. Until now, there is no technique showing real evidence of volumetric lifting. 

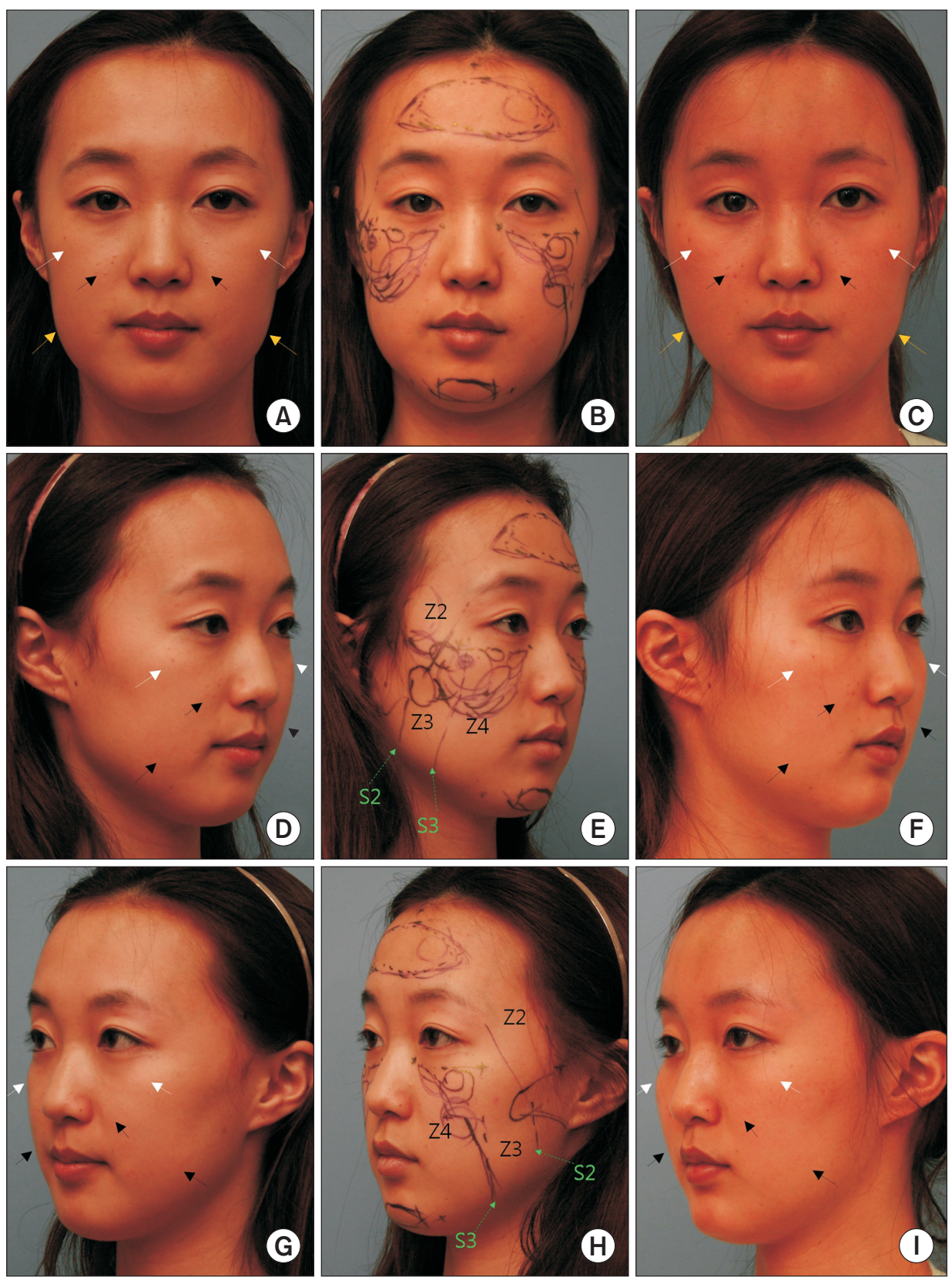

Fig. 2. A 27-year-old female with an asymmetrical face due to smaller and flatter malar bones on the right side of the face. The fat is injected into each side of the face (about $21.0 \mathrm{ml}$ on the right and $10.0 \mathrm{ml}$ on the left face) using the sequential autologous fat injection (SAFI) technique, except in the forehead and chin. (A, D, G) Preoperative photos; (B, E, H) Preoperative markings; (C, F, I) six months after fat grafting. White arrows: volume to be increased; black arrows: volume to be decreased; and yellow arrows: volume to be decreased with tightening of the sagging skin. Z, zone; S, SAFI line.
The author observed the lifting result by combining procedures that tightened the sagging jowls with decreased volume and shortened and smoothened the nasolabial folds with decreased volume, followed by the SAFI technique in the temporal, malar, lateral cheek, and submalar regions in all 3 cases. This effect was different from the fat rebalancing technique combined with fat grafting and liposuction.

As compared to the common results seen with a surgical facelift [9], the volume reduction in the nasolabial folds and jowls was the same; however, tightening and volume reduction near the incision lines was not observed with the SAFI technique. In fact, the volume increased and led the mid-face to appear small and round.

The result in Fig. 3 shows an example of pseudoptosis, where volume reduction caused the development of a deep nasolabial fold [10]. Moreover, it could be the same as the superolaterally lifted soft tissue drooping anteroinferiorly with the volume reduction of the grafted fat volume (Fig. 4C, D, G, H).

Unlike other fat grafting techniques [1-4,7,8], different volumes of purified fat were injected into each layer in a regular order in the SAFI technique, which led to the increase in the skeletal support for the overlying soft tissue. Eventually, this 

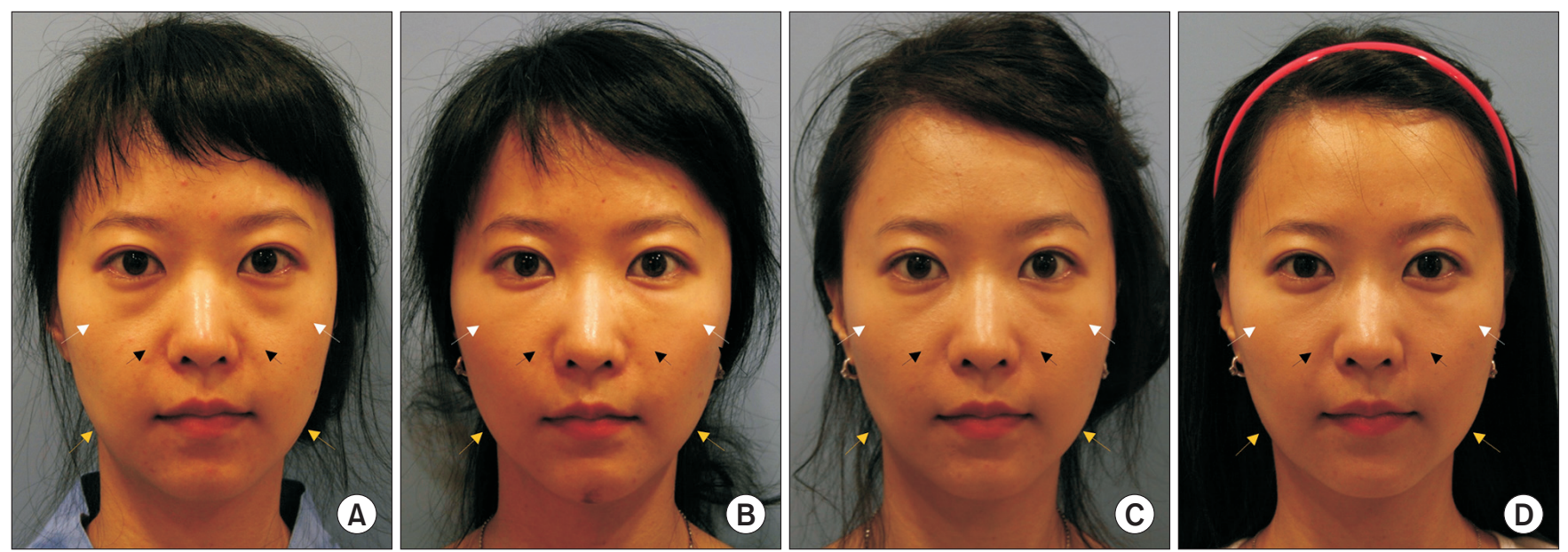

Fig. 3. A 26-year-old female underwent one session of fat grating for volume restoration. Total $45.0 \mathrm{ml}$ of fat was injected into the whole face by the sequential autologous fat injection (SAFI) technique. (A) Preoperatively; (B) two hours after procedure; (C) 3 months after procedure; (D) one year after procedure. White arrows: volume to be increased; black arrows: volume to be decreased; yellow arrows: volume to be decreased with tightening of sagging skin.
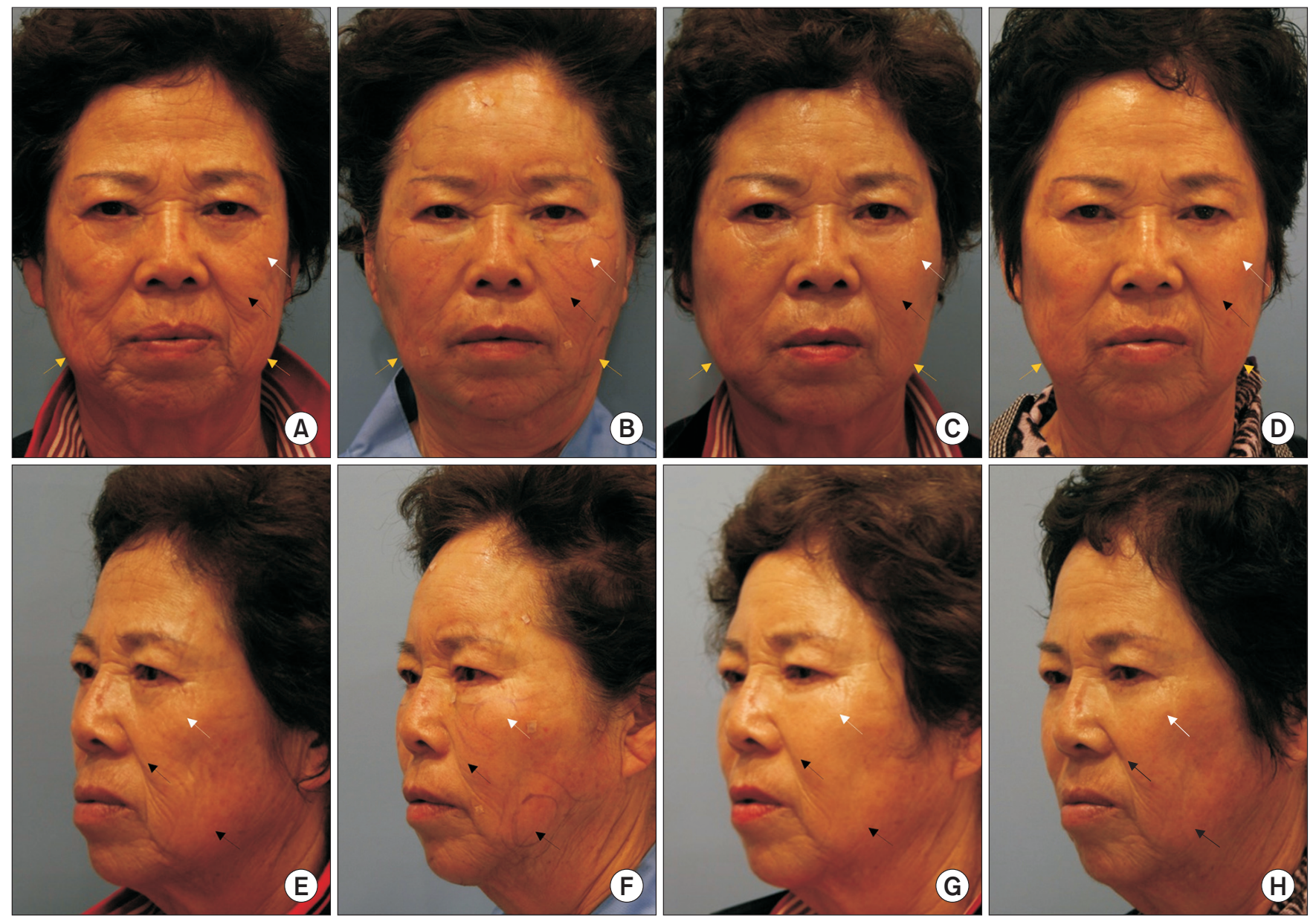

Fig. 4. A 64-year-old female underwent fat grafting procedure with 4 sessions of postoperative fractional laser. Total $76.0 \mathrm{ml}$ of fat was grafted into the entire face and forehead by the sequential autologous fat injection (SAFI) method. (A, E) Preoperatively; (B, F) immediately after the procedure; $(C, G)$ one month after the procedure; $(D, H)$ one year and 3 months after the procedure. White arrows: volume to be increased; black arrows: volume to be decreased; and yellow arrows: volume to be decreased with tightening of the sagging skin. 
seemed to be the main cause of volumetric lifting.

This is not enough evidence to conclude that the SAFI technique has a volumetric lifting effect with the results from these 3 cases, because the evaluated cases were small in number and limited to patients with small and flat malar bones. However, this report is considerably worthy of initial evidence on volumetric lifting using fat grafting technique. The SAFI technique results should be researched further through scientific analysis of a large number of clinical cases. The author observed an actual lifting effect by fat grafting in 3 patients with small and flat malar bones.

\section{Conflicts of interest}

The author has nothing to disclose.

\section{References}

1. Coleman SR. Structural fat grafting. Aesthet Surg J 1998;18: $386,388$.

2. Donofrio LM. Fat rebalancing: the new "facelift". Skin Therapy Lett 2002;7:7-9.

3. Amar RE, Fox DM. The facial autologous muscular injection (FAMI) procedure: an anatomically targeted deep multiplane autologous fat-grafting technique using principles of facial fat injection. Aesthetic Plast Surg 2011;35:502-10.

4. Lam SM, Glasgold MJ, Glasgold RA. Complementary fat grafting. Philadelphia (PA): Lippincott, Williams, \& Wilkins; 2007.

5. Kang KJ, Chai CY. Complementary effect of fat grafting on traditional subciliary incisional lower blepharoplasty: estimated by the change of lid-cheek junction. J Cosmet Med 2018;2:1221.

6. Kang KJ. SAFI: a novel facial grafting technique with a concept of volumetric lifting. Paju: Koonja; 2017. p. 197-248.

7. Sattler G. The tower technique and vertical supraperiosteal depot technique: novel vertical injection techniques for volume-efficient subcutaneous tissue support and volumetric augmentation. J Drugs Dermatol 2012;11:s45-7; discussion s47.

8. Rohrich RJ, Ghavami A, Constantine FC, Unger J, Mojallal A. Lift-and-fill face lift: integrating the fat compartments. Plast Reconstr Surg 2014;133:756-67e.

9. Chang S, Pusic A, Rohrich RJ. A systematic review of comparison of efficacy and complication rates among face-lift techniques. Plast Reconstr Surg 2011;127:423-33.

10. Rohrich RJ, Pessa JE, Ristow B. The youthful cheek and the deep medial fat compartment. Plast Reconstr Surg 2008;121:2107-12. 